\title{
Geographic Load Balanced Routing in Wireless Sensor Networks
}

\author{
Robin Guleria \\ IBM India \\ robinguleria@gmail.com \\ Ankit Kumar Jain \\ Lovely Professional University, India \\ jain_ankit2407@yahoo.com
}

\begin{abstract}
Recently the application domains of wireless sensor networks have grown exponentially. Traditional routing algorithm generates traffic related to route discovery to destination. Geographic routing algorithms exploit location information well but the problem of congestion and collision throttle its full employment for resource constrained wireless sensor networks. In this paper we present a Geographic Load Balanced Routing (GLBR), explores a technique Load balancing for WSNs which can be a viable solution to the challenges of geographic routing. Load balancing can be realized through two approaches. GLBR defines parameters based on communication overhead at sensor nodes and wireless link status through which load can be balanced across whole network. GLBR approach exploits the existing Geographic Routing approach i.e. Greedy forwarding by considering not only the distance between next hop and destination as single parameter for packet forwarding but also consider overhead at node. When load at a node is high GLBR looks for an alternate option for packet forwarding. Thus GLBR divert traffic to obviate congestion and hence avoid disconnections in the network.
\end{abstract}

Index Terms - Wireless Sensor Network, Routing protocol, Load balance

\section{INTRODUCTION}

Wireless sensor networks (WSNs) are increasingly becoming vital to the development of smart environments. These networks have applications in modern day systems, as they aid in the military operations, architectural constructions, industrial processes, Battlefield surveillance and home applications.

The sensors in WSNs are battery operated and batteries, in many instances, do not last longer periods and cannot be recharged so need for development of appropriate techniques which guarantee the longevity of WSNs is must.

For the wireless networks the communication requires traversal through multiple hopes. Till now various routing schemes have been investigated ranging from traditional routing algorithms to ad-hoc routing algorithms. With the traditional routing algorithms, problem generation of traffic related to finding the routes to the end point and also they require the full topological information of the network. Ad-hoc approaches for routing also face similar problem as they require either the full topological information of wireless networks or topological information of the routes to the destination, for finding the shortest path.

Geographic routing algorithms have been proved as a promising successor of ad-hoc routing techniques. Packet forwarding decision in Position based routing techniques depends upon the geographic distance between the nodes. It scale better, require less maintenance and lower route setup expenses. Though a number of Geographic routing algorithms have been proposed but they suffers with the problem that traffic routed to the same region always follow the same path. This greedy forwarding approach results in consumption of more power at particular nodes which sometimes results in disconnected network. It also leads to the congestion problem at a particular node.

We present Geographic Load Balanced Routing (GLBR) a noble approach for Routing the packet to end point. GLBR adapts Geographic routing and traffic information at each neighbor of the forwarding node for forwarding the packets. The packet will be forward to that neighbor node whose cost to deliver the packet is minimum. Thus GLBR try to disseminate the traffic across the whole network and therefore avoid congestion at a particular sensor node and hence prevent the disconnection of network.

Geographic routing algorithms have been proved as a promising successor of ad-hoc routing techniques. Originally it was believed that geographic face routing could ensure guaranteed packet delivery, but unfortunately, later it comes to knowledge that the greedy forwarding leads to early network disconnection. In Geographic routing traffic routed to the same region always follow the same path. This leads more power consumption at specific nodes, while other remained underutilize. So, with our algorithm GLBR we try to overcome this and increase network lifetime. 
The remainder of the paper is arranged as follows. Section-II provides the background about geographical routing and face routing in WSN. Various approaches to balance node in geographic routing present in sectionIII.The algorithm for balancing node is proposed in section-IV. Simulation framework along with various result followed by discussion has been reported in section-V. Based upon the simulation results, conclusions have been drawn and some recommendations for future work have been proposed in section-VI.

\section{RELATED WORKS}

In this section, provide a survey of previous Position based (Geographic) routing and its challenges. An overview of literature related to geographic routing is there and in the last we will conclude with the survey of other routing schemes for wireless networks.

\section{1- Geographic Routing}

The Initial proposals for geographic routing were simply based on greedy forwarding approaches [1, 2]. These algorithms even in a connected network did not guarantee a packet delivery and packet was being dropped. This is a case when greedy forwarding ends up at a local minimum.

Compass Routing II (face routing) was the first geographic routing scheme to guarantee packet delivery [3]. For choosing next hop it only use local information stored at current hop. At every hop in between this will continue until destination not meet. Compass Routing does not keep the track of those messages that passed through a particular node. Because it utilizes enough memory at nodes where traffic is high, which leads memory overload.

Since then several algorithms GPSR [4], GEAR [5] and the GOAFR+ algorithms [6] that are variations of compass routing have been proposed. In GPSR [4] If the router position is known and also the destination is known the node will communicate the packet to the neighbor being closest to packet's destination (Euclidean distance). As only neighbor and destination addresses are stored, rather than whole network, it is almost a stateless protocol. The problem of voids arises when the alone path to the destination requires a node to send packet to a farther location but temporarily.

GEAR [5] (Geographic and Energy Aware Routing) use energy aware neighbor selection for routing towards the target region. When the packet is in target region, simple flooding with duplicate suppression method is used. The recent inclusion to this series is GDSTR [7], which rather than constructing the planar graph, in case of voids, construct the Hull Tree.

\section{Challenges in Geographic Routing:}

Greedy Perimeter Stateless Routing (GPSR) due its small state storage requirements, robustness and low routing protocol overhead scales well for moving routers networks. It was believed that geographic face routing could ensure guaranteed packet delivery. But for practical radio networks the distributed planarization of network graphs is a serious challenging problem [8]. Radioattenuating obstacles introduce non-uniformity in radio ranges. The communication range of radio is strictly dependent on the environment and due to radioattenuating obstacles it may be highly irregular. This can cause planarization failure. For real environment obstacles the assumption of Unit Disk Graph is often violated [9].

Another assumption, that nodes knew their geometric positions and radio ranges accurately leads to errors in localization. In case of Practical Radio environment Kim et al. proposed CLDP (Cross-Link Detection Protocol)[10] which guarantees to work accurately for such conditions. They proposed right-hand rule to find out whether there are cross links or not in the network graph. CLDP is quite successful in detecting a planar graph correctly in practical conditions, but it has higher cost of probing single link multiple times.

Generally geographic routing works best when greedy forwarding is employed for packet forwarding and CLDP has high maintenance costs and complexities, so it is considerable to have another geographic routing algorithm which obviate planarization requirement. Ben Leong Proposed GDSTR [7], which rather than constructing the planar graph, in case of voids, construct the Hull Tree [7]. Thus overcome planarization cost and making geographic routing more practical.

What we propose is that as in geographical routing packet forward to the same destination always chose the same next hop, thus leading to high energy depletion at that particular node, which sometimes results in disconnected graph. Our approach tries to distribute the load across whole network and thus avoid load at a particular node and avoid the disconnections in the network.

\section{2- Face Routing}

Face Routing is the basis of entire work till now on geographic routing, which ensures Packet delivery in static connected plane graphs. This is very rare in wireless network. In face routing a network graph is being divided into a set of well defined faces. A node just needs to know about immediate neighbors. The packet is routed along these faces. When face routing starts the first route or edge to be traversed is in clockwise order around the initial vertex by using right-hand rule to traverse a face. The process will continue until destination reached. Packets contain information regarding its source and destination to detect non delivery and cycles. 


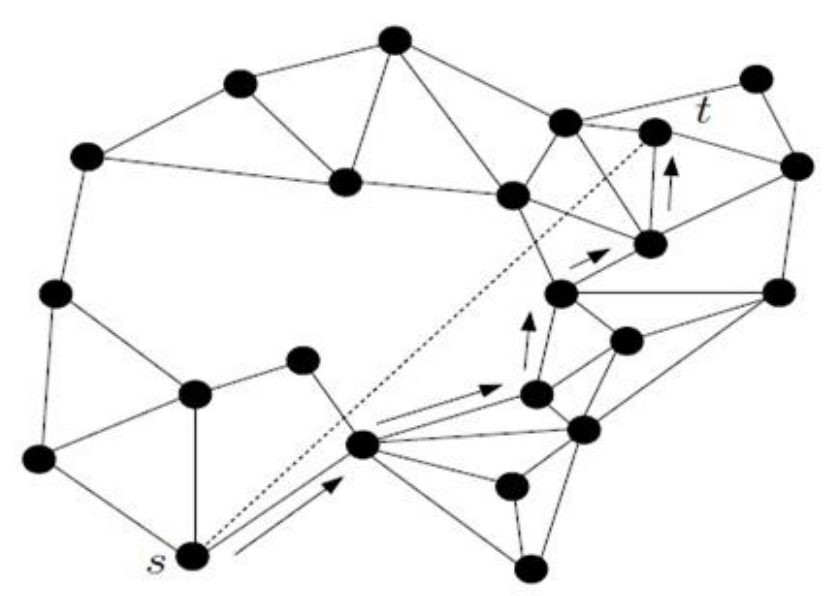

Figure 1: An example of the path taken between $\mathrm{S}$ and $\mathrm{t}$.

The figure-1 shows the faces that need to be traversed. Hence, for detecting that a packet at the boundary of a face and should cross over to traverse another face, node only needs to see if the line from $S$ to $t$ crosscut any of its outgoing edges. Face routing can fail when a packet loops along a sequence of faces. This is case when face traversal algorithm uses the any node as the new source rather than intersection point.

The existing face routing approaches differs by how they route the packets across the faces of planarized graph. These approaches have two constraints first for routing they need a spanning plane subgraph of the network and second they assume that during routing the plane subgraph remains static. GPSR [6] uses a deterministic right-hand rule when forwarding the packet along a face. Like the GPSR protocol, the GOAFR+ [6] protocol is also a combination of greedy forwarding and face routing. GOAFR+ [6] keeps a track of how far packet has gone along that face. It will backtrack if there is no progress towards destination. To pick the optimal forwarding direction GPVFR keeps several hops information.

\section{3- Network Planarization}

Network planarization is the foundation of GPSR, GOAFR and several other routing protocols. To find a planar subgraph that contains all the nodes of network is itself a challenge. Kranakis et al. only described a routing mechanism called face routing [3], but nothing was proposed for constructing planar sub-graphs. Planarization has been used in applications like topology discovery, data-centric storage, network localization and several others etc. The first step in planarization of a network graph is to choose optimal route both in terms of distance or hop between two vertices. The advantage of a shortest path is, in uniform radios such a path usually does not change.

Bose et al. proposed mechanism based on Gabriel Graph (GG) [19] for the planar sub-graph, where as Karp and Kung proposed using the Relative Neighborhood Graph (RNG) [4]. Planarization algorithms works efficiently for few restrictive models with certain assumption that the underlying network must be a unitdisk graph. But for more practical network models due to radio-attenuating obstacles the transmission ranges are not uniform. Another assumption, that nodes knew their geometric positions and radio ranges accurately leads to errors in localization. Therefore no efficient planarization algorithm is available.

\section{Relative Neighborhood Graph}

\section{$R N G$ can be defined as:}

"For a set of vertices with known positions, edge $(a, b)$ exist between $a$ and $b$ if only there does not exist any other point c, known as witness vertex, closer to both a and $b$ than distance between them. While removing make sure that graph is not disconnected at any stage. "

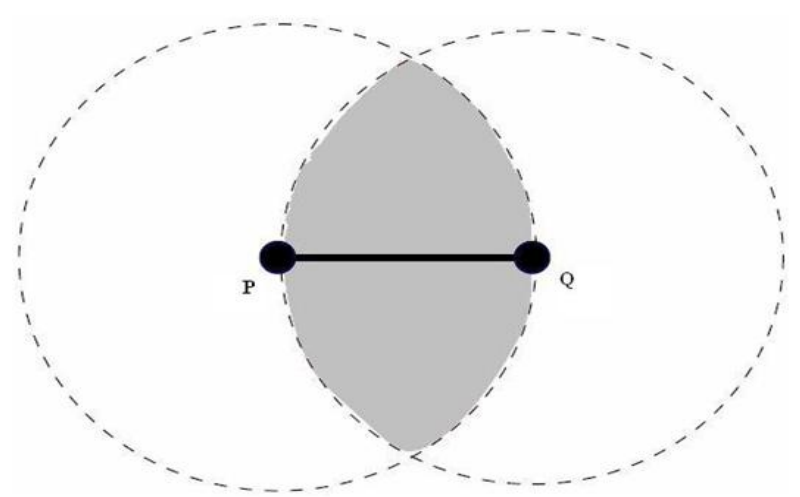

Figure 2. Relative neighbor Graph

The intersection is called the relative neighborhood or lune. So for edge $(p, q)$ there should not be any witness in shaded region.

\section{Gabriel Graph}

Gabriel Graph searches a smaller shaded region for a witness node when compared with the RNG.

\section{The GG is a superset of RNG. GG can be defined as:}

"Two elements are Gabriel neighbors, sphere with diameter as distance between them does not contain any other points. The GG is a subset of the Delaunay triangulation."

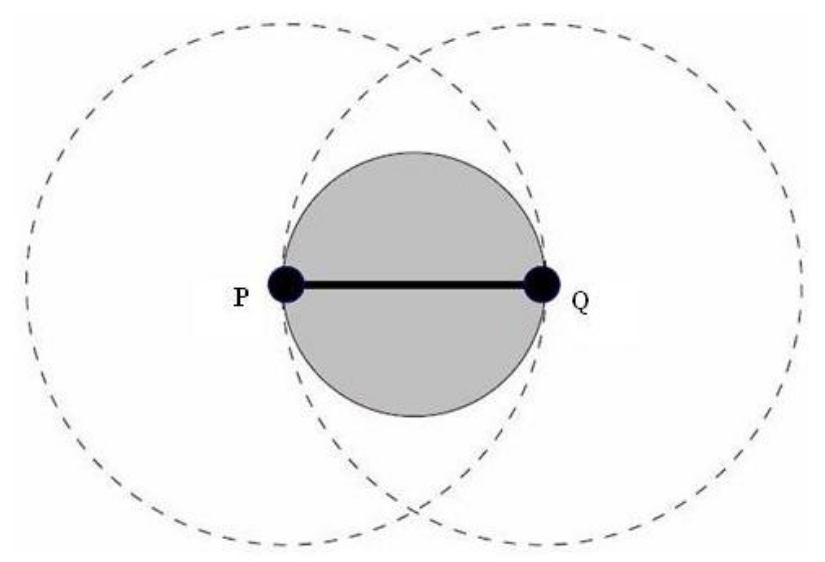

Figure 3 Gabriel Graph 
For edge $(\mathrm{p}, \mathrm{q})$ to be included shaded sphere should not contain any witness node. Because the Gabriel neighborhood is contained in the relative neighborhood, it has no witness node when the latter doesn't have. Few other distributed algorithms like the Restricted Delaunay Graph and Localized Delaunay Graph that produce planar graphs have also been proposed.

Kim et al. examine network challenges in practical scenario and proposed CLDP. Though CLDP [10] is able to planarize an arbitrary graph but its high cost is something needs to be taken care. To make graph planar they proposed that in a graph every node should independently probe each of its links by a right-hand rule. Cross-Link Detection Protocol (CLDP) [10], for practical radio networks where radio-attenuating obstacles poses different problems like localization error and transmission range is not uniform, produces a sub-graph that work correctly.

\section{APPROCHES TO BALANCE NODE IN GEOGRAPHIC ROUTING}

This section describes the approaches through which we can achieve load balancing in case of geographic routing in WSNs.

To reduce the complexity in wireless/mobile networks a number of location based routing protocols [3-7], has been proposed. Rather than topological information they rely on geometric location of nodes. They are conceived as good candidate for networks with limited computing capability nodes, like WSNs, to deliver information. These protocols are almost stateless as they require little information to be stored at each node. They require destination location and next hop neighbors' location for packet forwarding. These protocols are regarded as better working protocols than traditional ones [23, 25, 27].

Though this approach relishes several advantages, like little control overheads, no frequent updates and are suitable for battery-powered sensor nodes of WSNs, this technique suffers collision and congestion. So when it comes to the constrained resources in wireless sensor networks geographic routing protocol plays major role, therefore there is need of some modifications into original greedy forwarding that can enhance its efficiency and can improve network lifetime.

In geographic routing nodes situated at the crossing of multiple paths that leads to sink are engaged in more packet handling, so will consume more resources and run out its energy and this more often results in partitions in network. Furthermore, forward packets to a specific node in next hop results in collision and network congestion. So load balancing for energy constrained sensor networks is vital.

Two factors for load balancing can be derived from properties (limited available bandwidth, disconnections etc.) of WSNs. First, is communication overhead at node and other is wireless link status. The former is related with overheads expected to be induced at each node whereas the later with the status of wireless link between adjacent nodes. Thus, parameters are derived from node data itself and are very critical for communication.

a)Parameters for communication overhead

\section{b) Parameters for wireless link status}

\section{A) Communication Overhead:}

Communication overhead is related to how much overheads or load is expected to induce at any node. The information regarding communication overhead is inferred from the node itself. Node under high stress means more traffic and therefore congestion is likely to occur. Communication overhead can be measured efficiently by four different parameters. The first is average number of packets that are received between a predetermined time periods. More reception means more overhead. The next total number of neighbors is a considerable factor to see how much stress (traffic) the node will suffer or is under. Third is based on energy. More energy expenditure means more overhead. And last parameter is number of entries in current routing table on each node.

\section{The average number of packets received:}

Each node has a finite memory. Number of packets the node was engaged in routing are stored at each node. This parameter gives higher priority to actual task (i.e. the actual packet routed during communication). If the count is higher than a threshold limit means node is under high stress and will suffer collision and congestion.

\section{The total number of neighbors:}

Node's stress level depends on the neighbor count of a particular node. Obviously the node located in a dense deployment is expected to get engage in more packet transmission i.e. handling request and responses than sparse deployment. Total number of neighbors, other way of defining it is node degree i.e. with how many nodes it is in a direct communication. Thus, communication overhead level will vary in accordance with the denseness of network deployment.

\section{Energy Level of a node:}

Remaining battery level can be a good signal of high communication overhead. More energy expenditure signifies that node was busy in handling more request and responses. Therefore third parameter for deciding communication overhead is related with energy level of a node. As mostly, all sensor nodes start their operations with same battery level initially, therefore low battery level at any moment indicates that this node is under high stress and will most probably die soon than others.

Number of entries on current routing table of each node:

The third parameter is more specialized than previous two and is based on number of routing entries on each node. Entries determine with how many nodes to communicate or overhead to decide next hop. More entries means the load at the node is building up and to tackle congestion there is need of load balancing. 


\section{B) Wireless Link Status:}

It is related to current link status between communicating nodes. Low quality wireless medium can lead to severe problems on the communication and in any network is very critical, so parameters indicating Wireless link status can be considered for balance load in the network.

\section{Average retransmission time:}

Time needed for transmitting the message completely over the link. Wireless links are quite unstable and communication is not so reliable over these links. Packet delivery ratio shows reliability on the link partly. So for reliable communications over wireless medium retransmission schemes are generally being used to recover lost packets. An undelivered message requires retransmission. Retransmission schemes ensure delivery of transferred message. So, there is an alternative parameter for link Status, average transmission time. Both queuing delay and retransmission make this value longer.

\section{Average packet delivery ratio:}

It reflects success ratio of transmission and also link characteristics. It is ratio of total delivered packets to destination. High delivery ratio indicates that link is reliable and can transmit the more packets without error. Therefore, performance of network is also measured in terms of probability of packet loss.

By defining these parameters we can combine it with original greedy forwarding scheme. For packet forwarding we will not consider the distance as the only parameter but will also use above defined parameters.



Figure 4. Parameters to balance Load

\section{METHODOLOGY}

In this section we will explain the Geographic Load Balanced Routing (GLBR) algorithm. We will also explain certain assumption considered in this work and calculation of cost to deliver packet to destination.
In Geographic routing greedy forwarding is employed, that may lead to collision, congestion and also sometimes network disconnections. In our proposed solution routing is not done alone on shortest distance between destination and next hop. Now for packet forwarding both the greedy approach and traffic at nodes will also be considered. In GLBR, among the load balancing approaches we choose the number of packets received in predetermined time parameter for distribute the load. We keep track of traffic that passed through each node. Depending on the load or overhead at each node we will switch to a better alternate node.

\section{A. Assumption}

For simulating GLBR we consider certain assumptions. We assume that all wireless sensor nodes know their positions through a GPS device or by other localization techniques. Geographic Load Balanced Routing (GLBR) assumes that nodes are capable of sending beacons to neighbors. Beacons contain information regarding traffic levels of node. The distance between base station and node is also broadcasted. Further topologies considerations are such where the network is deployed in a plane. Whether a link exists between sensor nodes or not is determine by distance between them and are bidirectional. Nodes are in range if distance between them is below certain threshold. Finally, we assume that packet destination is known to all packet sources. This information assists in selection of next hop for packet forwarding and is stored at node's cache.

\section{B. Algorithm for GLBR}

The implementation of the GLBR algorithm is described into three different steps. First is the network deployment. Second is to calculate the cost of nodes to deliver packet to destination. Third is the description of GLBR algorithm.

\section{Network Deployment}

a) $\mathrm{N}$ numbers of WSN nodes are deployed randomly within a certain boundary area within a 2Dimensional plane. $\mathrm{N}$ may vary from some hundred nodes to thousands nodes.

b) A node can communicate up to a maximum distance determined by a transmission range. So a transmission range is specified for each node. And further links are bidirectional in nature.

c) A node is neighbor of another node if they are separate by a distance less than their transmission range. This distance can be calculated in 2-D coordinate system by using the distance formula. If $\left(\mathrm{u}_{1}, \mathrm{u}_{2}\right)$ are coordinate of one node and $\left(\mathrm{v}_{1}, \mathrm{v}_{2}\right)$ are coordinate of second node, then distance between them is:

$$
D=\sqrt{\left(u_{2}-u_{1}\right)^{2}+\left(v_{2}-v_{1}\right)^{2}}
$$

\section{Cost Function Calculation:}

For choosing next hop GLBR also consider cost of a node to deliver packet, which is a function of distance 
[5]. We will show that for different network densities our GLBR shows high packet delivery ratio. Our main focus will be on static network.

We will simulate our GLBR using ns2. We will measure the performance of GLBR by using 2 metrics network lifetime and average packet delivery ratio. We will deploy 50, 100 and 200 nodes into an area of $1000 \mathrm{~m} * 1000 \mathrm{~m}$. Beacon interval for GLBR is 10 seconds. We will run the simulation till the first node in the network dies and that defines network life time for GLBR. In this simulation each source node annotates packets with location of the destination.

In GLBR all packets are destined to base station. We will evaluate GLBR using two metrics Network Lifetime and Packet Delivery Success Ratio. We investigate effect of density on GLBR performance varying node degree from 6 to 10 per node on average.

Energy model: For calculating the energy expenditure of transmitting the packet we will use the first order radio model [30]. According to first order radio model transmission energy for $\mathrm{k}$ bits is

$$
\operatorname{ETX}(\mathrm{k} ; \mathrm{d})=\operatorname{elec} * \mathrm{k}+\varepsilon \mathrm{amp} * \mathrm{k} *
$$

And for receiving $\mathrm{k}$ bits is

$$
\operatorname{ERx}(\mathrm{k})=\text { Eelec } * \mathrm{k}
$$

Where $E_{\text {elec }}=50 \mathrm{nj} / \mathrm{bit}$ and $\varepsilon_{\mathrm{amp}}=100 \mathrm{pJ} / \mathrm{bit} / \mathrm{m}^{2}$

For GLBR Load level will depend on the Number of packets received in one second. Values of $L_{1}, L_{2}$ and $L_{3}$ are shown in table 1.

Table 1: Load levels at nodes

\begin{tabular}{|c|c|}
\hline Load level & $\begin{array}{l}\text { Number of packets received in one } \\
\text { second }\end{array}$ \\
\hline $\mathrm{L}_{1}$ & Less than 3 \\
\hline $\mathrm{L}_{2}$ & Greater than or equal to 3 and less 6 \\
\hline $\mathrm{L}_{3}$ & Greater than or equal to 6 \\
\hline
\end{tabular}

\section{B. Network Deployment}

The deployment of 100 nodes is shown in figure in an area of $1000 \mathrm{~m} * 1000 \mathrm{~m}$. Base station location is fixed at coordinate $(500,100)$. And all packets are annotated with base station location.

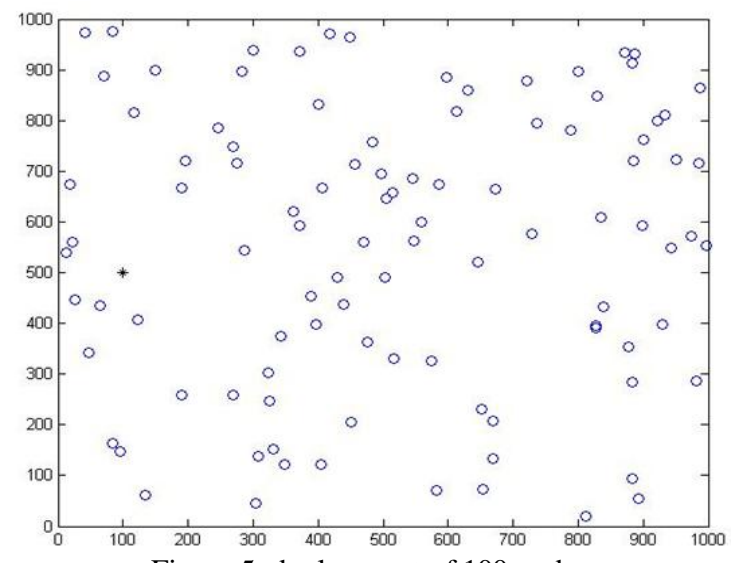

Figure 5: deployment of 100 nodes

\section{Performance Evaluation}

Network lifetime for GLBR is defined as when a first node in the network dies.

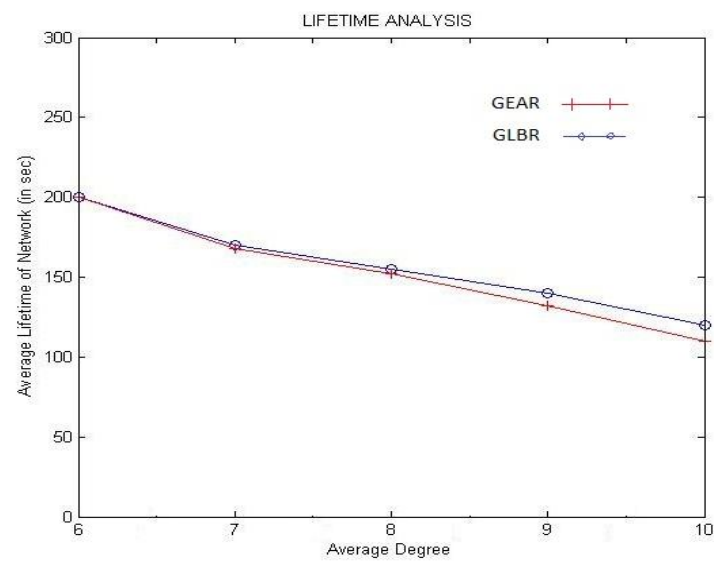

Figure 6: Comparison of lifetime of the GLBR and GEAR

When we compare the lifetime of our GLBR with GEAR we found that when the node degree is around 6 and 7 the GLBR performance when compare to GEAR is similar. But as the density of network increases more and more packets are being flooded into the networks. In this case GLBR behaves well as compare to GEAR because when the node degree is high nodes are going to receive more packets and as GEAR only consider energy to decide next hop it will suffer collision. This is where GLBR performs better. GEAR obviates packet drops at nodes due to congestion and saves energy drainage at node and thus increases lifetime. GLBR has an upperhand over GEAR because in regions where a nodes' energy is not utilized enough as it is not routing packets but all of sudden node experience sudden activities, GEAR in this scenario will suffer collision but as GLBR analyzis traffic before forwarding packets it will choose an alternate.

\section{Packet Delivery Success Ratio:}

The second metric is packet delivery success ratio. In addition to prolonging the network lifetime, GLBR assists nodes to deliver more packets, therefore increases network performance. On an average GLBR delivers almost $96 \%$ of the packet sent. When the node degree increases GLBR doesn't show a sharp fall in packet delivery ratio. When traffic on particular nodes starts building up GLBR employ load balancing and looks for another alternate obviating congestion and collision. The graph in figure shows with the increase in node degree the performance of GLBR doesnot got affected too much. GLBR while calculating Packet Delivery Success Ratio include only those packets for which route exist to destination. Failure due to disconnected destination are not covered in GLBR. 


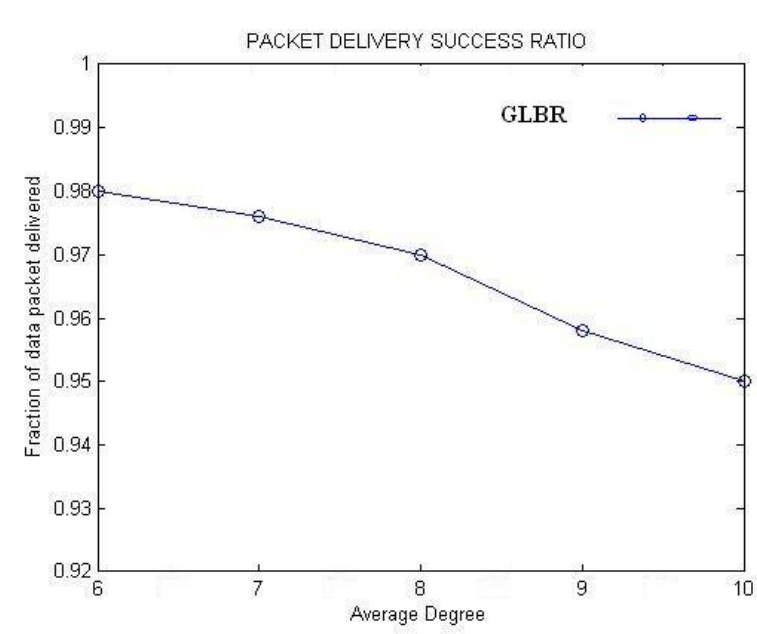

Figure 7: Packet delivery success ratio of GLBR

Thus simulation results have shown that GLBR outperforms GEAR when the node density is high. GLBR delivers almost $96 \%$ of the packets.

\section{CONCLUSIONS AND FUTURE WORK}

The real motivation behind geographic routing is that state maintained at nodes is almost negligible and therefore is less expensive than traditional routing algorithms to deliver packet. We have presented Geographic Load Balanced Routing, GLBR, a geographic routing algorithm that uses energy and traffic at nodes to achieve load balancing on densely deployed wireless networks. Our algorithm when compared to GEAR shows better results when density of network increases. We in this work addresses greedy forwarding limitations when packet destined to destination always follow same path causes stress at some nodes leads to energy drainage which results in disconnections. GLBR handle collision and congestion in greedy forwarding well. This strategy obviates early network disconnections in traditional greedy forwarding. We have proposed parameters based on communication overhead and wireless link status to realize load balancing. State requirement at nodes is proportional to neighbors and is quite less than traditional adhoc approaches. Simulation shows that GLBR consistently delivers $96 \%$ packets successfully on an average.

Our objective is to explore new ideas through this work. GLBR handles geographic routing challenges (collision and congestion) in networks where sensor nodes are immovable. In future we want to implement GLBR for networks where nodes are movable as application domains (medical care and disaster management) of WSNs are continuously increasing. Accuracy of mobility in geographic networks depends on the accuracy of the underlying localization mechanism. So, location estimation in GLBR for introducing mobility is itself a challenge. Due to time constraint introducing mobility in this work is difficult but in future it can be realized.

\section{REFERENCES}

[1] Tingh-Chao Hou and Victor O.K. L, "Transmission range control in multihop packet radio networks," IEEE Transactions on Communications, 34(1), pp. 38-44, 1986.

[2] Gregory G. Finn, "Routing and addressing problems in large metropolitan scale internetworks," Technical Report ISI/RR-87-180, ISI, March 1987.

[3] E. Kranakis, H. Singh, and J. Urrutia," Compass routing on geometric networks," In Proceedings of the 11th Canadian Conference on Computational Geometry, pp 51-54, August 1999.

[4] B. Karp, and H. T. Kung," GPSR: Greedy perimeter stateless routing for wireless networks," In Proc. ACM/IEEE MobiCom, pp 243 - 254, Aug. 2000.

[5] Y. Yu, D. Estrin, and R. Govindan," Geographical and Energy-Aware Routing: A Recursive Data Dissemination Protocol for Wireless Sensor Networks," UCLA Computer Science Department Technical Report, UCLA-CSD TR-01-0023, May 2001.

[6] F. Kuhn, R. Wattenhofer, and A. Zollinger. "WorstCase Optimal and Average-Case Efficient Geometric Ad-Hoc Routing", In Proceedings of 4th ACM International Symposium on Mobile Ad Hoc Networking and Computing (MobiHoc 2003),pp 267 - 278 June 2003.

[7] Ben Leong, Barbara Liskov, and Robert Morris "Geographic Routing without Planarization", In Proceedings of the 3rd conference on NSDI 2006 - Volume 3 pp. 25 - 25,2006.

[8] Brad Karp. Challenges in geographic routing: Sparse networks, obstacles, and traffic provisioning, May 2001.

[9] Y.J. Kim, R. Govindan, B. Karp, and S. Shenker "On the pitfalls of geographic face routing", In Proceedings of DIAL-M-POMC 2005, pp. $34-43$ September 2005.

[10] J. Kim, R. Govindan, B. Karp, and S. Shenker "Geographic routing made practical", In Proceedings of NSDI 2005, pp. 217--230, May 2005.

[11] JOHNSON, D. B., AND MALTZ, D. B. "Dynamic source routing in adhoc wireless networks. In Mobile Computing", T. Imielinski and H. Korth, Eds. Kluwer Academic Publishers, ch. 5, pp. 153$181,1996$.

[12] L. Dooley, I. Gondal, and M. Iqbal, "An energytime based load balancing technique for wireless sensor networks," in Intelligent Sensors, In Proceedings of the 2005 International Conference on Sensor Networks and Information Processing, pp 57-62, 2005.

[13] I. Akyildiz, W. Su, Y. Sankarasubramaniam, and E. Cayirci, "A survey on sensor networks," IEEE Commun. Mag., pp. 102-114, Aug. 2002.

[14] M. Gerla, and S. Lee,'Dynamic load-aware routing in ad hoc networks," in Communications, 2001. ICC 2001. IEEE International Conference on," vol 10 ,pp 3206-3210, 2001. 
[15] A. Argyriou, and V. Madisetti, "Realizing loadbalancing in ad-hoc networks with a transport layer protocol," In Proc. of Wireless Communications and Networking Conference, 2004, IEEE., pp. 18971902, March 2004.

[16] Le Zou, Mi Lu, and Zixiang Xiong, “A Distributed Algorithm for the Dead End Problem of Location Based Routing in Sensor Networks,'In Proc. Of Transactions on Vehicular Technology, vol. 54, no. 4, July 2005.

[17] Guoliang Xing, Chenyang Lu, Robert Pless, and Qingfeng Huang," On greedy geographic routing algorithms in sensing-covered networks," In Proceedings of the 5th ACM International Symposium on Mobile Ad-Hoc Networking and Computing (MobiHoc 2004), pages 31.42, 2004.

[18] B.Baranidharan, B.Shanthi, "A Survey on Energy Efficient Protocols for Wireless Sensor Networks, "International Journal of Computer Applications (0975 - 8887) Volume 11- No.10, December 2010.

[19] Prosenjit Bose, Pat Morin, Ivan Stojmenovic, and Jorge Urrutia. "Routing with guaranteed delivery in ad hoc wireless networks," Wireless Networks, 7(6):609.616, 200118.

[20] Jie Gao, Leonidas J. Guibas, John Hershberger, Li Zhang, and An Zhu, "Geometric spanner for routing in mobile networks, " In Proc. of the 2001 ACM Symposium on Mobile AdHoc Networking and Computing (MobiHoc 2001), pages 45.55, July 2001.

[21] S Radhakrishnan, Gopal Racherla, Chandra N. Sekharan, N. S.V Rao, and S.G. Batsell. DST, "Arouting protocol for ad hoc networks using distributed spanning trees, "In IEEE Wireless Communications and Networking Conference, 1999.

[22] Rodrigo Fonseca, Sylvia Ratnasamy, Jerry Zhao, Cheng Tien Ee, David Culler, Scott Shenker, and Ion Stoica. "Beacon vector routing: Scalable pointto-point routing in wireless sensor nets," In Proceedings of the 2nd Symposium on Networked Systems Design and Implementation (NSDI 2005), May 2005.

[23] Charles Perkins and P. Bhagwat "Highly dynamic destination-sequenced distance-vector routing (DSDV) for mobile computers," In Proceedings of ACM SIGCOMM'94 Conference,pp 234-244, August 1994.

[24] M. Mauve, J“org Widmer, and Hannes Hartenstein. "A survey on position-based routing in mobile ad hoc networks," IEEE Network Magazines, 15(6), November 2001

[25] David B Johnson and David A Maltz, "Dynamic source routing in ad hoc wireless networks," In Mobile Computing, volume 353, 1996

[26] Charles Perkins, “Ad-hoc on-demand distance vector routing," In Proceedings of IEEE Military Communications Conference (MILCOM '97), November 1997.

[27] Zygmunt J. Haas, "A routing protocol for the reconfigurable wireless networks," In IEEE International Conference on Universal Personal Communications (ICUPC'97), volume 2,pages 562.566, 1997.

[28] Wendi Rabiner Heinzelman, Anantha Chandrakasan, and Hari Balakrishnan,"Energy Efficient Communication Protocol for Wireless Microsensor Networks," In Proceedings of the 33rd Hawaii International Conference on System Sciences 2000.

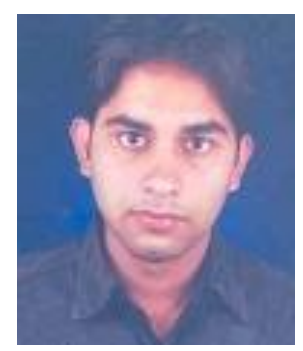

Ankit Kumar Jain received the Master of technologyin Information Technology with specialization in Wireless communication and computing from Indian Institute of Information Technology Allahabad, India. He is presently working as Assistant Professor in Lovely Professional University, Punjab, India.

$\mathrm{He}$ is highly engaged in teaching and research. His general research interest is in the area of wireless Networks, Wireless Sensor Network and Mobile computing. He has published many papers in reputed journals and conferences.

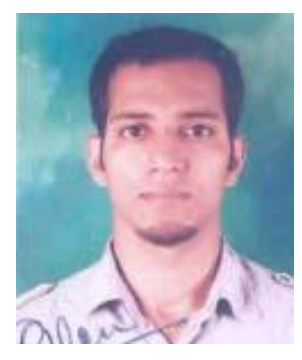

is in the area Computer Networks.
Robin Guleria is Associate System Engineer in IBM, India. He received the Master of technology in Information Technology with specialization in Wireless communication and computing from Indian Institute of Information Technology Allahabad, India. His general research interest of Wireless Sensor Network and 